\title{
ARTICLE \\ PD-1 is expressed by and regulates human group 3 innate lymphoid cells in human decidua
}

\author{
Paola Vacca', Silvia Pesce ${ }^{2}$, Marco Greppi ${ }^{2}$, Ezio Fulcheri ${ }^{3,4}$, Enrico Munari ${ }^{5,6}$, Daniel Olive ${ }^{7}$, Maria Cristina Mingari ${ }^{2,8}$, \\ Alessandro Moretta ${ }^{2}$, Lorenzo Moretta ${ }^{1}$ and Emanuela Marcenaro ${ }^{2}$
}

\begin{abstract}
Group 3 innate lymphoid cells (ILC3) have been detected in both murine and human decidual tissues where they are thought to play a relevant role in the induction and maintenance of pregnancy. However, limited information exists on the molecular mechanisms that regulate these cells, including immune checkpoints. Here, we show that ILC3 express the inhibitory checkpoints programmed cell death (PD-1) and T cell immunoglobulin and mucin domain containing protein 3 (TIM-3) during the first trimester of pregnancy and that these receptors could regulate production of cytokines, including IL-22, IL-8, and TNF-a, induced by IL-23. We also show that the intermediate extravillous trophoblast (iEVT) expresses high levels of the PD-1-ligand PD-L1, suggesting that PD1/PD-L1 interaction may regulate ILC3 function at the feto-maternal interface. Our present data provide the first evidence that human decidual ILC3 express a functional PD-1. It is possible that an altered expression or function of PD-1 may break the immunetolerance resulting in pregnancy failure.
\end{abstract}

Mucosal Immunology (2019) 12:624-631; https://doi.org/10.1038/s41385-019-0141-9

\section{INTRODUCTION}

Innate lymphoid cells (ILCS) belong to a family of immune cells involved in innate host defenses against pathogens and tumors, in lymphoid organogenesis, and in tissue remodeling/repair. Recently, ILCs have been classified into cytotoxic and helper. ${ }^{1}$ Natural Killer (NK) cells, representing cytotoxic-ILC, secrete primarily interferon- $\gamma$ $($ IFN- $\gamma$ ) and tumor necrosis factor- $a$ (TNF- $a$ ), and are responsible for innate defenses against tumors and viruses. ${ }^{2,3}$ NK cells also participate in the complex network of cell-to-cell interactions that leads to the induction of adaptive immune responses. ${ }^{3-6}$ Helper ILCs are further subdivided into three subsets on the basis of their transcription factor profile and cytokine production. Among helperILCs, ILC3s are RAR-related orphan receptor gamma t ${ }^{+}\left(\mathrm{ROR}_{\mathrm{t}}{ }^{+}\right)$and secrete interleukin(IL)-22, IL-17A, IL-8, and TNF-a. In adults ILC3s consist of two subpopulations that are commonly distinguished on the basis of the expression of natural cytotoxicity receptors (NCRs), a feature common to NK cells. In particular, CD56 ${ }^{+} \mathrm{ILC} 3$ express high levels of NKp44 and medium levels of NKp46, whereas CD56 ILC3, also referred to as lymphoid tissue inducer(LTi)-like cells, actually express low levels of NKp44 and may or may not express NKp46.? NK cell function is under the control of inhibitory checkpoints, that may be constitutively expressed such as the human leukocyte antigen (HLA)-I specific killer-cell immunoglobulin-like receptors (KIRs) and CD94/NKG2A ${ }^{8}$ or inducible such as programmed cell death (PD-1). ${ }^{9}$ On the other hand, limited information exists on checkpoint receptors expressed by ILC3s. Both NK cells, displaying peculiar properties, and ILC3s are present in human decidua where they are thought to play a relevant role in neo-angiogenesis, tissue remodeling, and placentation as well as in the balance between inflammation and tolerance. ${ }^{10,11}$ However, the events that induce such critical balance have not been elucidated so far.

In the present study, we show that in the early phase of pregnancy, decidual ILC3s express functional PD-1 (and T-cell immunoglobulin and mucin domain containing protein 3 (TIM-3), while the invading trophoblast expresses the PD-1 ligand, PD-L1. This suggests that the PD-1/PD-L1 molecular interaction may play a regulatory role at the feto-maternal interface.

\section{RESULTS}

ILC3s expressing PD-1 and TIM-3 are present in human decidua Multiparametric flow cytometric analysis (Supplementary Fig. 1A, B) revealed that, in human decidua, both $\mathrm{CD} 56^{+} \mathrm{ILC} 3 \mathrm{~s}$ (NKp44 ${ }^{\text {high }}$, NKp46 ${ }^{+}$) and LTi-like cells (NKp44 low, NKp46 low) (Supplementary Fig. 2) brightly express PD-1 in variable proportions (Fig. 1a, b). In particular, higher percentages of $\mathrm{PD}-1^{+}$cells were detected in the LTi-like population. In contrast, decidual NK cells were PD-1 ${ }^{-}$ (Fig. 1a, b). In addition, ILC3s expressed the TIM-3 checkpoint, present in higher percentages in $\mathrm{CD} 56^{+} \mathrm{ILC} 3 \mathrm{~s}$ when compared to LTi-like cells (Fig. 1c, d). Notably, a fraction of ILC3s coexpressed

\footnotetext{
${ }^{1}$ Department of Immunology, IRCCS Bambino Gesù Children's Hospital, Rome, Italy; ${ }^{2}$ Department of Experimental Medicine (DIMES) and Centre of Excellence for Biomedical

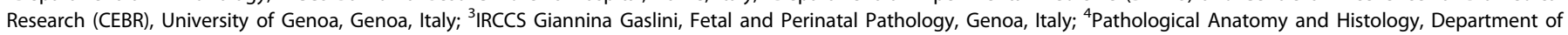

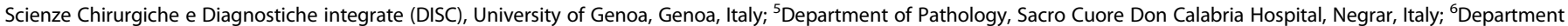

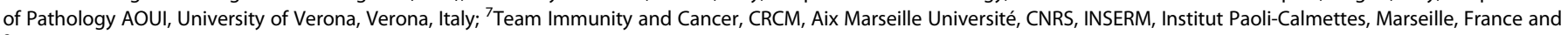
${ }^{8}$ UOC Immunologia, IRCCS Ospedale Policlinico San Martino Genova, Genoa, Italy

Correspondence: Lorenzo Moretta (lorenzo.moretta@opbg.net) or Emanuela Marcenaro (emanuela.marcenaro@unige.it)

These authors contributed equally: Paola Vacca, Silvia Pesce. These authors contributed equally: Lorenzo Moretta, Emanuela Marcenaro.

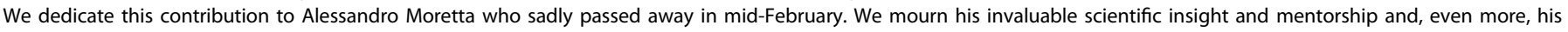
humanity, irony and smile.

Received: 7 August 2018 Revised: 22 December 2018 Accepted: 23 January 2019

Published online: 12 February 2019 
a

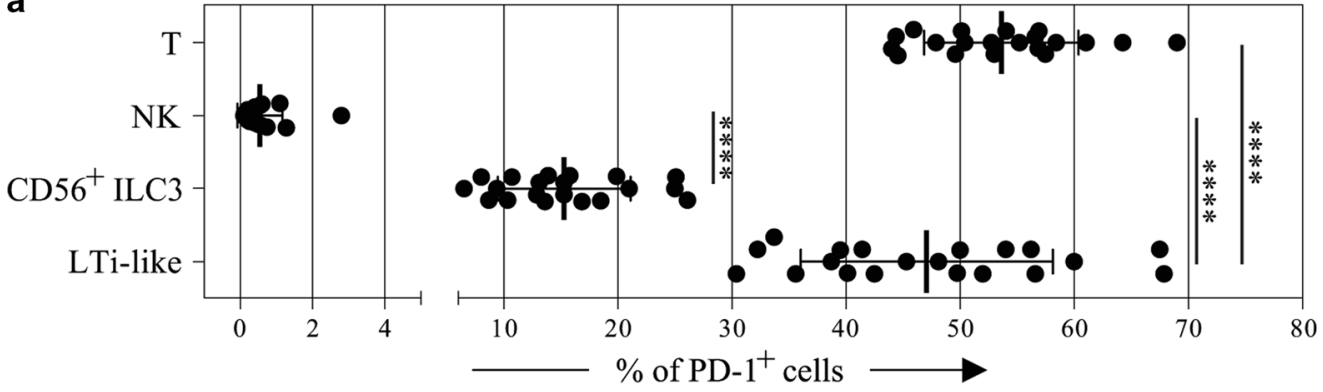

b
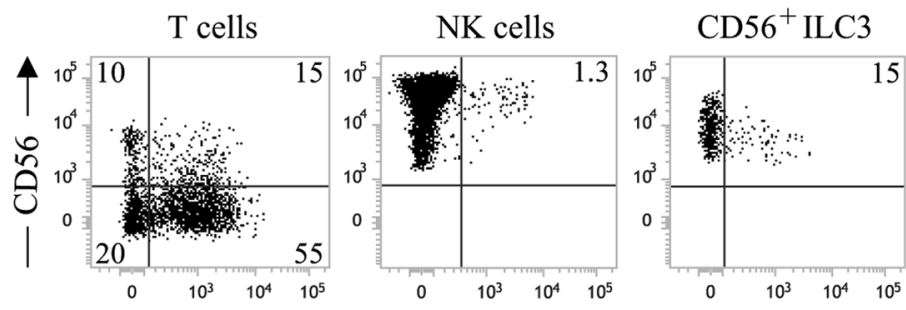

LTi-like

PD-1

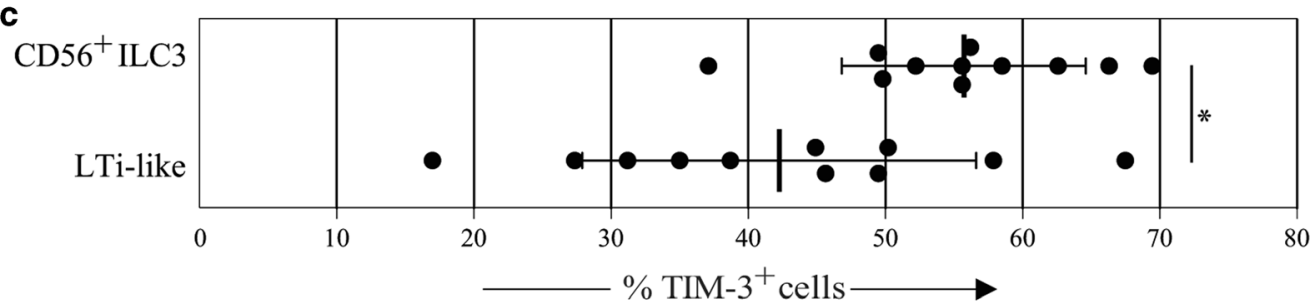

d

Donor 1
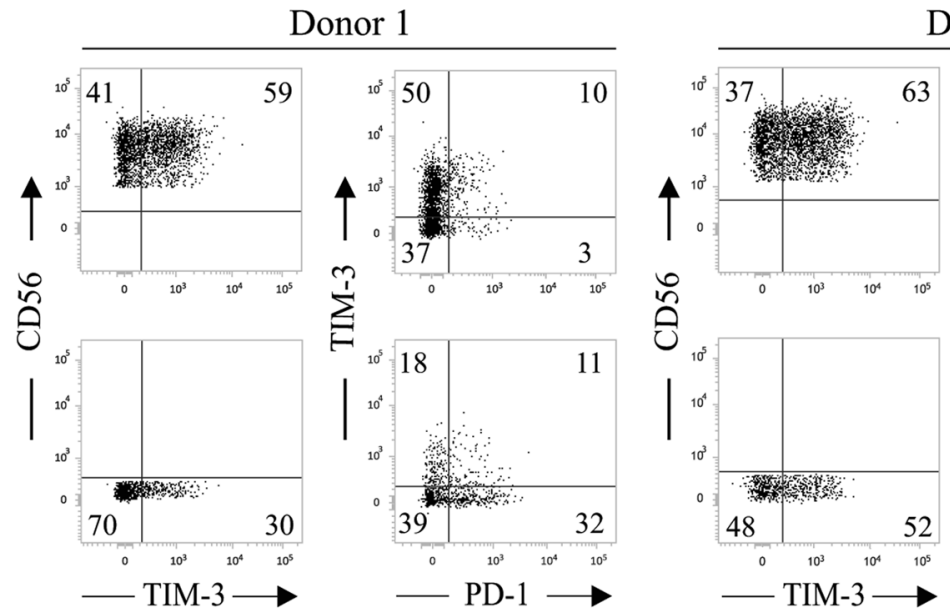

Donor 2

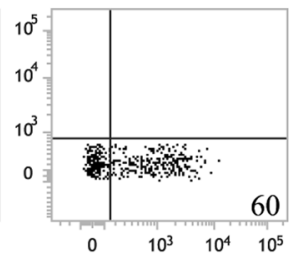

e
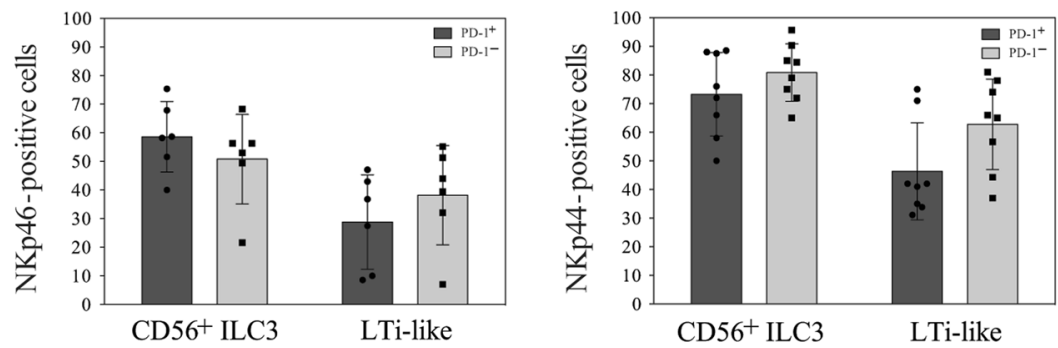

Fig. 1 PD-1 and TIM-3 phenotypic analysis on decidual-infiltrating lymphocytes isolated from first-trimester decidual tissues. a Percentages of PD $-1^{+}$cells in the indicated lymphoid populations (data represent the mean \pm SD; $n=20$ ). $\mathbf{b}$ PD-1 expression in one representative donor is shown. c Percentages of TIM- $3^{+}$cells in the indicated lymphoid populations (data represent the mean \pm SD; $n=11$ ). $\mathbf{d}$ TIM-3 expression and coexpression with PD-1 on CD56 ${ }^{+}$ILC3 and LTi-like cells in one representative donor is shown. e Coexpression of PD-1 with NCRs (NKp46 and NKp44) on CD56 ILC3 and LTi-like cells. NCR: natural cytotoxicity receptor 
TIM-3 and PD-1, while variable proportions expressed either TIM-3 or PD-1 (Fig. 1d). Other immune checkpoints, including LAG-3 and TIGIT, were virtually negative while KLRG1 was present in small percentages only in some donors (Supplementary Fig. 1C). Moreover, in order to exclude that LTi-like cells $\left(\mathrm{CD} 56^{-} \mathrm{CD} 117^{+}\right)$ may include some ILC2s $\left(\mathrm{CD} 56^{-} \mathrm{CD} 117^{+} \mathrm{KLRG} 1^{+}\right)$, we assessed the presence of a typical ILC2 marker, namely CRTH2. ${ }^{12}$ As shown in Supplementary Fig. 1C, both ILC3 subsets did not contain $\mathrm{CRTH}^{+}$cells.

We further characterized the phenotypic profile of ILC3s expressing PD-1 and/or TIM-3, in order to correlate the expression of these immune checkpoints with other markers and with potential given functional features. To this end, we analyzed PD $-1^{+} / \mathrm{PD}-1^{-}$or TIM- $3^{+} / \mathrm{TIM}^{-3^{-}}$ILC3 subpopulations for the expression of NKp46 and NKp44 activating NK receptors and of cytokine/chemokine receptors, including IL-15Ra (IL-15 is a cytokine physiologically present in the decidual microenvironment ${ }^{13}$ ), IL-23R (IL-23 is a cytokine involved in ILC responses), and CCR6 (allowing to identify ILC3 subset). Regarding the activating NK receptors, we observed a lower expression of $\mathrm{NKp} 46$ on $\mathrm{PD}-1^{-}$as compared to PD-1 ${ }^{+}$ CD56 ${ }^{+}$ILC3s, while the expression trend of NKp46 was opposite on LTi-like cells. Instead, the expression of NKp44 was higher in PD-1 than PD- $1^{+}$both in $\mathrm{CD} 56^{+} \mathrm{ILC} 3 \mathrm{~s}$ and in LTi-like cells (Fig. 1e). In addition, we observed that both $\mathrm{PD}-1^{+} / \mathrm{PD}-1^{-}$or $\mathrm{TIM}-3^{+} / \mathrm{TIM}-3^{-}$ subsets homogeneously expressed CD117 and CD127, two typical ILC3 markers (data not shown). Regarding cytokine/chemokine receptors, we did not detect any difference in terms of IL-15 Ra, IL-23R and CCR6 expression in both PD- $1^{-}$and PD-1 ${ }^{+}$ILC3 subsets (data not shown). These results are in line with our previous study ${ }^{10}$ showing that decidual ILC3 subsets (including CD56 ${ }^{+}$ILC3s and LTi-like cells) homogeneously express high levels of these receptors. These data suggest that, in decidual tissues, ILC3s expressing or not PD-1 do not display significant differences in their ability to respond to specific cytokines.

PD-1 and TIM-3 expressed by human decidua ILC3s are functional ILC3s present in decidua are thought to contribute to tissue building/remodeling thanks to their capability of producing proinflammatory cytokines/chemokines and of recruiting neutrophils. ${ }^{14}$ However, a normal pregnancy requires also to dampen excessive inflammation and to establish a tolerogenic status at the feto-maternal interface. In this context, we investigated whether PD-1 could affect the cytokine production by PD- $1^{+}$ILC3s induced by overnight incubation with IL-23, IL1 $\beta$, and IL-7. Thus, by applying a reverse antibody-dependent cellular assay, we could detect a significant reduction of cytokine production upon PD-1 monoclonal antibody (mAb)-mediated cell triggering in both ILC3 subsets. In particular, as shown in Fig. 2a, mAb-mediated cross-linking of PD-1 resulted in a significant reduction of cytokine production by PD $-1^{+} \mathrm{CD} 56^{+} \mathrm{ILC} 3 \mathrm{~s}$. On the other hand, anti-PD1 $\mathrm{mAb}$ did not affect the cytokine productions of $\mathrm{PD}-1^{-}$ CD56 ${ }^{+}$ILC3s. A significant reduction of cytokine production could be detected also in PD- $1^{+}$, but not in PD- $1^{-}$LTi-like cells upon cross-linking of PD-1 (Fig. 2b). The level of cytokine production was further increased by the addition of anti-NCRs mAbs in both ILC3 subsets. Also in this case, the addition of anti-PD-1 mAbs inhibited the cytokine production (Fig. 2a, b). Similar results were obtained upon TIM-3 cross-linking. In particular, mAb-mediated cross-linking of TIM-3 resulted in a significant reduction of IL-22 production by $\mathrm{CD} 56^{+} \mathrm{ILC} 3 \mathrm{~s}$ (Fig. 2c).

Further functional experiments indicated that ILC3s can be inhibited also by physiological interactions occurring between PD1 and PD-L1 (using recombinant PD-L1), although to a lower extent as compared to anti-PD-1 mAb cross-linking. This result confirms our hypothesis that the PD-1/PD-L1 axis may indeed control decidual ILC3s (Supplementary Fig. 3).

Taken together, these data suggest that PD- 1 and TIM-3 may be involved in the regulation of ILC3 function during the first trimester of pregnancy. Indeed, during this early phase, both inhibition of inflammation and tolerance induction are required to avoid immune-mediated fetal rejection.

Intermediate extravillous trophoblast (iEVT) cells express PD-L1 To further document the possible involvement of the PD-1 inhibitory pathway in feto-maternal tolerance, we investigated whether PD-L1, the major PD-1 ligand ${ }^{15}$ was expressed in decidual tissues. It is of note that, during early pregnancy, iEVT cells, although expressing paternal HLA-antigens (in particular HLA-C), do not elicit immune responses by maternal T and/or NK cells. Interestingly, as shown in Fig. 3a, the iEVT cells brightly express PD-L1 while, moving away from the embryonic pole, the number of iEVT cells is drastically decreased together with PD-L1 expression. Moreover, low levels of PD-L1 are expressed also in decidual stromal cells (Fig. 3a, b) and CD14 ${ }^{+}$myelomonocytic cells (Fig. 3b). We repeated these analyses on 13 samples. These data would support the notion that ILC3s may favor the immunetolerance at the feto-maternal interface through the involvement of the PD-1/PD-L1 pathway. In this context, we also analyzed the PD-L1 expression in iEVT cells derived from five spontaneous abortions (during the first trimester) characterized by pathological decidual tissues (e.g. endometriosis cases, decidual trombosis). As shown in Fig. 3C, PD-L1 was expressed at much lower levels in these samples than in iEVT from normal pregnancies.

PD-1 expression is downregulated in ILC3s in the third trimester of pregnancy

We also analyzed ILC3s in the late phases of pregnancy (third trimester). The proportion of total ILC3s and NK cells among decidual infiltrating leukocytes (identified as $\mathrm{CD} 45^{+}$cells) were significantly decreased during term gestation (Supplementary Fig. 4A). Further analysis revealed that the percentage of PD-1 ${ }^{+}$ cells decreases in decidual ILC3s during preterm (32-41 weeks of gestation) reaching a minimum at term gestation (Fig. 4a, b). In contrast, the proportion of PD- $1^{+}$NK cells significantly increased during pregnancy (Fig. 3a, b and Supplementary Fig. 4B). These data were further substantiated by statistical analysis showing an indirect correlation between expression of PD-1 on ILC3s (in particular on LTi-like cells) and the stage of pregnancy (Fig. 4c, lower panel). On the other hand, a direct correlation exists between the percentages of PD-1 ${ }^{+}$decidual NK cells and stage of pregnancy (Fig. 4C, upper panel). Although not shown, unlike PD1 , the expression of TIM-3 remained substantially unmodified during pregnancy.

\section{DISCUSSION}

Our present study provides the first evidence that human ILC3s express functional a PD-1 receptor. In particular, in the case of decidual ILC3s, it is conceivable that PD-1 may play a regulatory role during pregnancy. Indeed, functional analysis revealed that cross-linking of PD-1 results in a significant functional inhibition of both $\mathrm{CD}_{56}{ }^{+} \mathrm{ILC} 3 \mathrm{~s}$ and LTi-like cell subsets leading to a decreased cytokine production. In addition to PD-1, high proportions of ILC3s expressed TIM-3, another immune checkpoint, that may also contribute to the regulation of ILC3s. Our data also suggest that the functional activity of PD- $1^{+}$ILC3s may be influenced by the interaction with embryonic trophoblast cells, expressing high levels of PD-L1. In this context, PD-L1 expression is primarily confined to iEVT cells (which are abundant in the first phase of pregnancy while they decrease at later stages). These data suggest that maternal immune cells, including different subsets of ILC3s, may be regulated by $\mathrm{PD}-\mathrm{L} 1^{+}$cells and contribute to the maintenance of feto-maternal tolerance.

Importantly, PD-1 expression on decidual ILC3s is maximal during the first trimester, i.e. the most critical period of pregnancy 
a

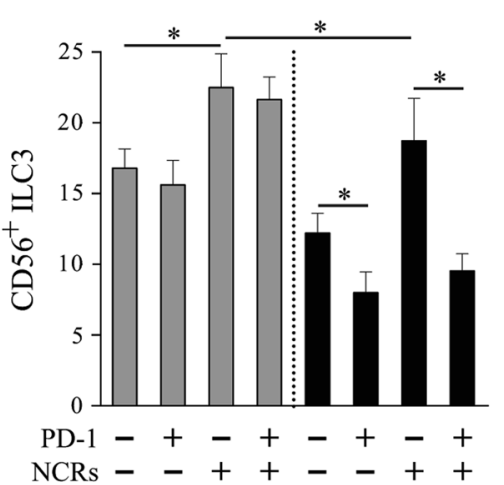

IL-8

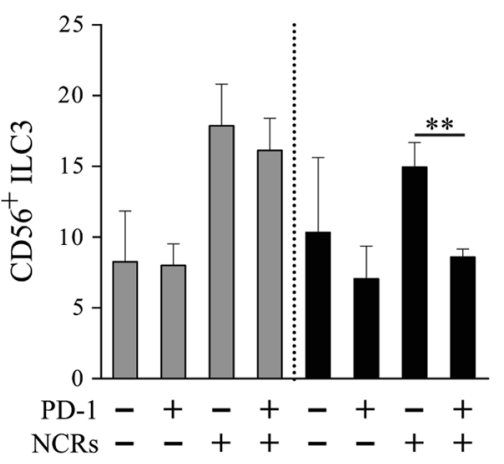

TNF- $\alpha$

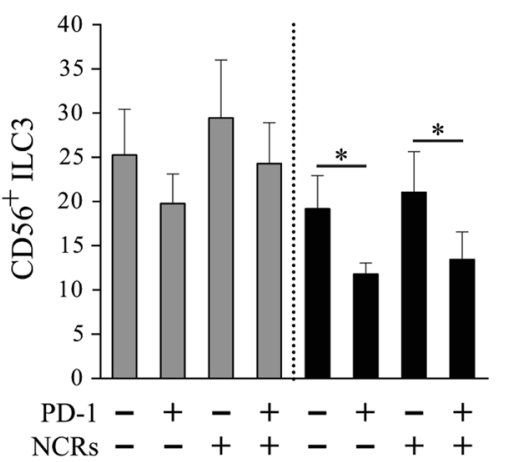

$\square$ PD-1- 1 b

IL-22

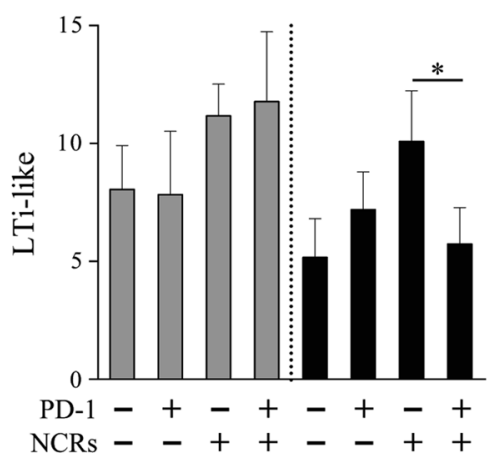

IL-8

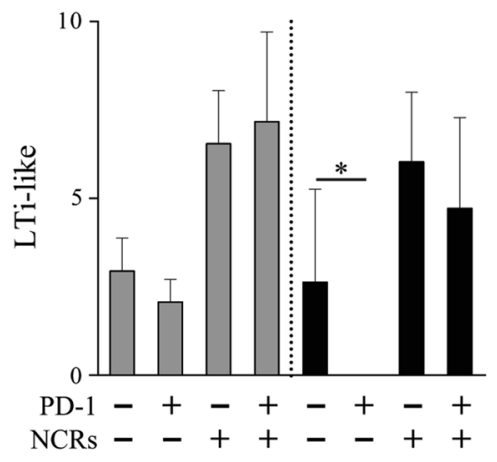

TNF- $\alpha$

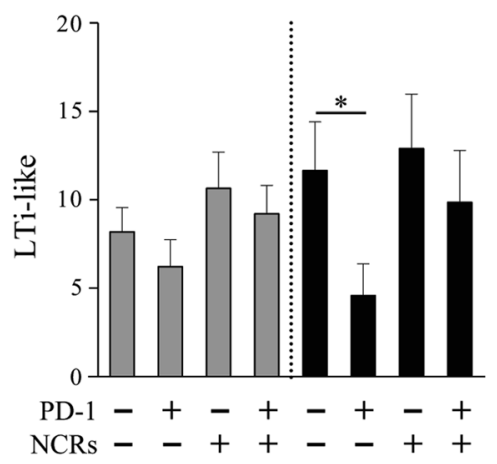

c IL-22
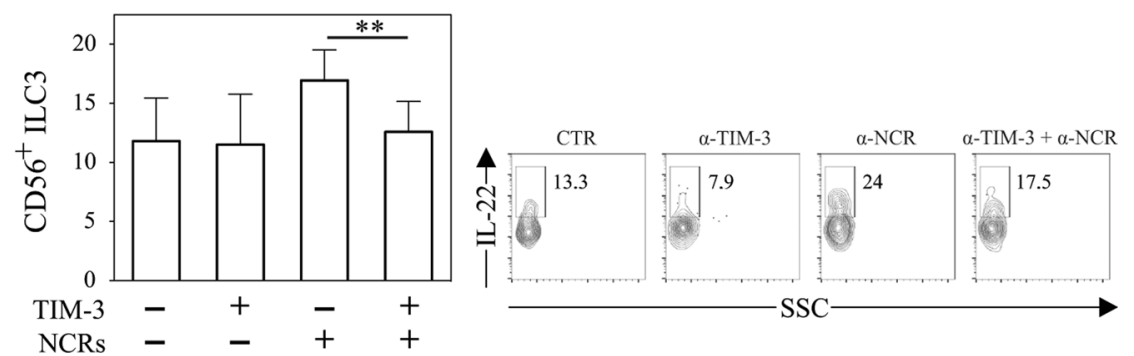

Fig. 2 Functional analysis of PD-1 and TIM-3 expressed in decidual-infiltrating ILC isolated from first-trimester decidual tissues. Intracellular cytokine detection by PD- $1^{-}$(gray bars) or PD- $1^{+}$(black bars) in a CD56 ${ }^{+} \mathrm{ILC} 3 \mathrm{~s}$ and $\mathbf{b}$ LTi-like cells after stimulation with different combinations of anti-NCRs and PD-1 (data represent the mean \pm SEM; $n=8$ ). ${ }^{*} P<0.05 ;{ }^{*} P<0.01$. c Intracellular IL-22 detection in CD56 ${ }^{+}$ILC3s after stimulation with different combinations of anti-NCRs and TIM-3 (data represent the mean \pm SEM; $n=8$ ). ${ }^{*} P<0.05$; ${ }^{* *} P<0.01$ (left). One representative donor is shown (right). ILC innate lymphoid cell, SEM standard error of the mean, NCR natural cytotoxicity receptor 

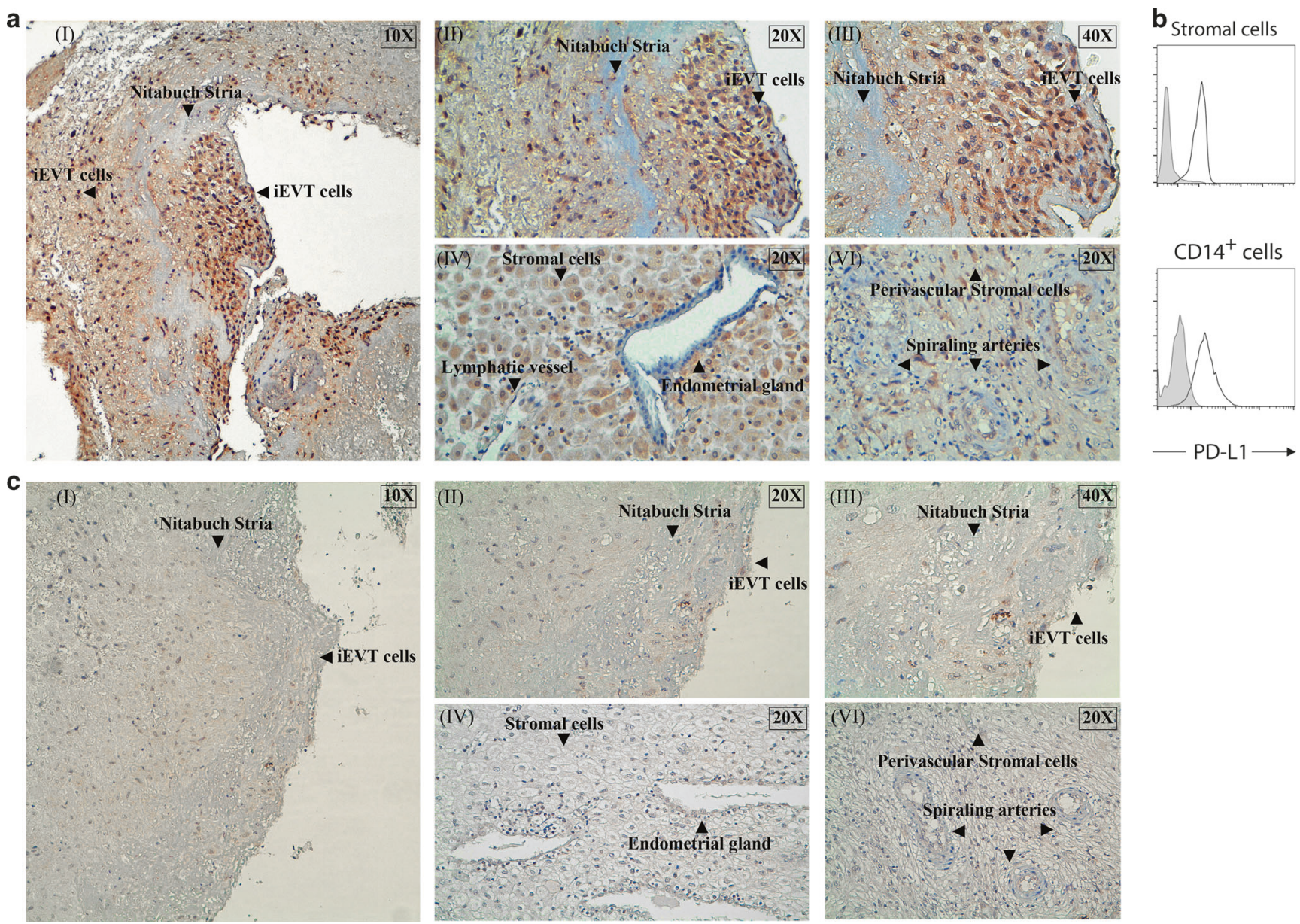

Fig. 3 Analysis of decidual tissues for PD-L1 expression during first trimester. a Bright immunolabeling of PD-L1 (brown cells) was present in intermediate extravillous trophoblast (iEVT) cells (arrow) forming clusters at the embryonic pole of a normal decidua (near the Nitabuch Stria). Moving away from the embryonic pole, the number of iEVT cells and PD-L1 staining decreases. Magnification at $\times 10$ (I), $\times 20$ (II), $\times 40$ (III) is shown. A weak positivity was also detected on stromal cells $\times 20$ (IV, V). Negative controls are represented by lymphatic vessel endothelium (IV), endometrial gland epithelium (IV), muscular tunic of spiraling arteries (V), stromal infiltrating lymphocytes (V). b PD-L1 expression on stromal cells and $\mathrm{CD}_{14}{ }^{+}$cells derived from decidual tissue during first trimester. c Negative immunolabeling of PD-L1 was detected in decidual tissue derived from pathological sample. Magnification at $\times 10$ (I), $\times 20$ (II), $\times 40$ (III) is shown

requiring efficient dampening/downregulation of the initial inflammatory phase, whereas it significantly decreases at later stages. This suggests an immune-regulatory role of PD- $1^{+} \mathrm{ILC} 3 \mathrm{~s}$ during the early phase of pregnancy. On the contrary, unlike PD-1, the expression of TIM-3 remained substantially unmodified.

The decrease of PD-1 expression during pregnancy is paralleled by a decrease in proportions of ILC3s among CD45 ${ }^{+}$cells. In this context, it has recently been demonstrated that an increased proportion of ILC2 and ILC3 occurs in decidua basalis and decidua parietalis (respectively) in women undergoing spontaneous preterm labor. ${ }^{16}$

On the other hand, during pregnancy, PD-1 expression significantly increases in decidual NK cells. A possible interpretation of these data is that, in the first trimester, NK cells, lacking PD-1, are kept under control by the interaction between their HLAspecific inhibitory receptors and HLA-C, HLA-G, and HLA-E molecules present on iEVT cells. ${ }^{17,18}$ In the third trimester, given the decrease of HLA-I antigens on trophoblast, ${ }^{18}$ an efficient control of NK cell function may require other checkpoints, such as PD-1. It is conceivable that an altered expression/function of PD-1 or PD-L1 may compromise the control exerted by this checkpoint, thus leading to the break of tolerance, possibly resulting in early pregnancy failure. In this context, our analysis of iEVT cells derived from spontaneous abortions occurring during the first trimester revealed low/negative expression of PD-L1. However, further investigation is needed to clarify to what extent maternal immune cells may be influenced by embryonic trophoblast to maintain fetal tolerance.

Thus, the balance between inflammation and tolerance is critical for reproductive success during placentation in the firsttrimester decidua. In this period, multiple ligand-receptor interactions can occur between trophoblast cells and decidual immune cells. These interactions are likely to control both villous syncytiotrophoblast, involved in nutrient exchange, and EVT cells, which invade decidua and remodel it. The immune microenvironment of the decidua prevents inflammatory responses that could potentially be triggered by trophoblast invasion and destruction of the smooth muscle media of the spiral arteries by trophoblast.

In decidua, ILC3s were shown to exert innate defenses (by releasing proinflammatory cytokines) and to contribute to tissue building/remodeling, thus favoring embryo implantation. These phenomena involve, at least in part, innate immune cells, particularly abundant in decidual tissues. Along this line, it has been shown that the cross-talk between decidual ILC3s and neutrophils is crucial for inducing the early inflammatory phase and to guarantee innate maternal defenses against pathogens. ${ }^{14}$ The inflammatory phase is followed by a regulatory phase 
a

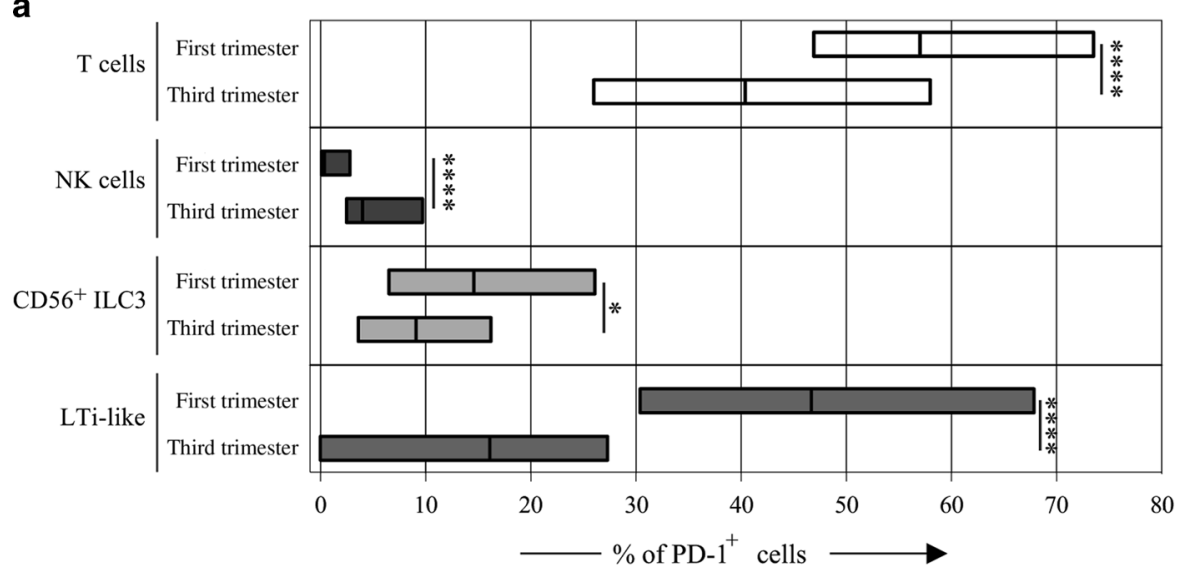

b

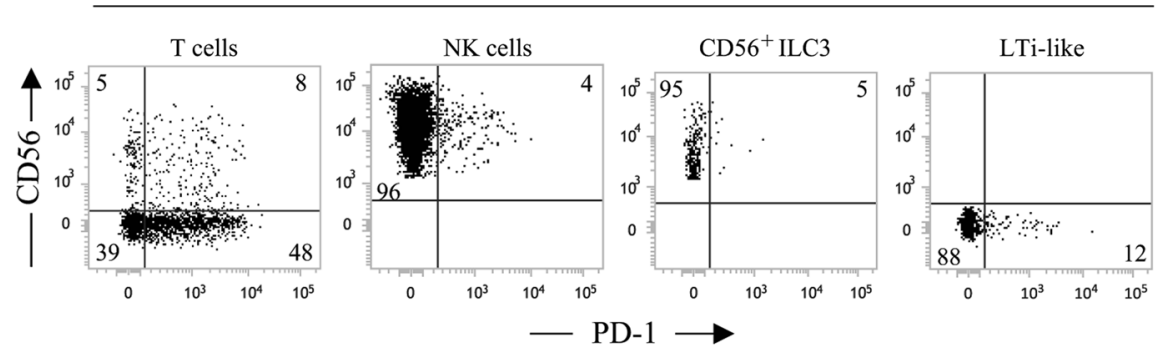

C

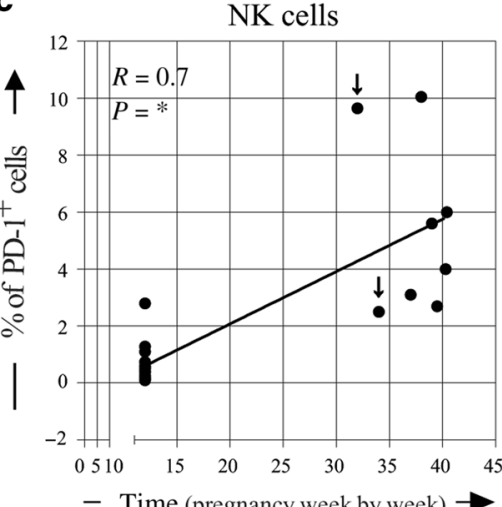

- Time (pregnancy week by week)

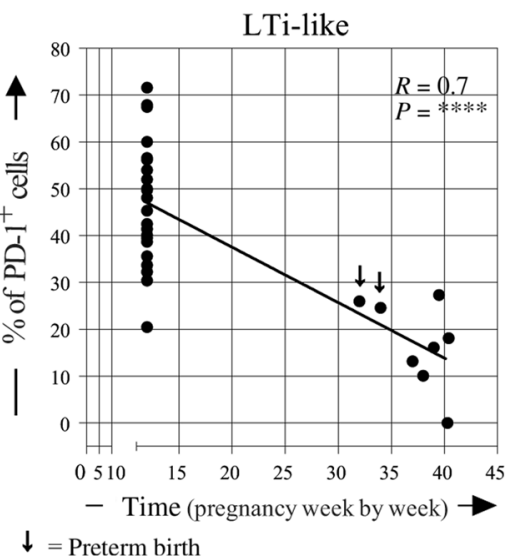

Fig. 4 Analysis of PD-1 on decidual-infiltrating lymphocytes from third trimester decidual tissues. a Comparison of PD-1 expression in T, NK, CD56 ${ }^{+}$ILC3 and LTi-like cells isolated at the first $(n=20)$ or the third trimester of pregnancy $(n=10)$. Data represent the mean \pm SD. $\mathbf{b} A$ representative donor is shown. c Correlation between proportion of PD $-1^{+}$NK cells or PD-1 ${ }^{+}$LTi-like cells and the pregnancy timing $(n=20$ first trimester decidual tissues; $n=8$ third trimester decidual tissues). The correlation was analyzed according to a linear regression model and confirmed by analyzing the Pearson Correlation Coefficient $(R=0.7)$. Arrows indicate preterm birth samples. ${ }^{*} P<0.05{ }^{* * * *} P<0.0001$

characterized by an increase in Tregs. ${ }^{19}$ These cells control the excessive inflammation and play a role in preventing immunemediated fetal rejection. In addition, the expression of inhibitory receptors (including immune checkpoints) on cytotoxic cells contributes to modulate/suppress specific immune responses. In this context, different checkpoints, including PD-1 and TIM-3, were detected on decidual $T$ lymphocytes in humans and on decidual $\mathrm{T}$ and NK cells in mice, suggesting their involvement in fetomaternal tolerance. ${ }^{20-22}$ Indeed, the expression of PD-1 on $\mathrm{CD}^{+}$ $T$ cells resulted in sharp decrease of their cytolytic activity and in the development of a Th2-dominant milieu in the decidua, an essential event to maintain normal pregnancy. ${ }^{23}$ The expression of inhibitory checkpoints on decidual immune cells further supports the notion that, in decidua, potentially damaging maternal immune responses to fetal trophoblast cells are under tight control. ${ }^{24}$ Indeed, our study suggests the existence of putative inhibitory interactions between ILC3s, expressing PD-1 and/or TIM3 , and EVT that express high levels of PD-L1. In particular, functional analysis revealed that, upon antibody-mediated crosslinking of PD-1 (or TIM-3), or PD-L1 engagement of PD-1, ILC3s display a reduced ability to release cytokines including IL-22, IL-8, and TNF-a (and IL-17A). It is conceivable that ILC3-mediated release of proinflammatory cytokines may exert an innate antimicrobial activity, thus contributing to defenses against infectious agents. However, to allow maintenance of pregnancy, it is necessary to dampen excessive inflammatory immune responses and to establish/induce a tolerogenic status at the feto-maternal interface.
It has been shown that PD-L1 expression could affect responses against self and foreign antigens, while the disruption of PD-1/PD$L$ interactions may allow to unleash $T$ and NK cell responses against tumors. Indeed, signals delivered by PD-1 represent a major mechanism of cancer immune escape. We show that in women experiencing a miscarriage during the first trimester (due to different pathological conditions), the expression of PD-L1 on iEVT cells was lower than in normal pregnancy. Thus, since PD-1 blockade may allow to control tumors and immune diseases by modulating PD-1 signal, it is possible to speculate that during the early phase of pregnancy, PD-1/PD-L1 interaction may play an important role in the control of the inflammatory phase, by preventing an excessive inflammation that could cause fetal rejection. All these results further confirm the notion that the PD1/PD-L1 axis may control decidual ILC3s. In this context, an altered expression/function of PD-1 or PD-L1 may result in the break of tolerance, possibly causing early pregnancy failure. Therefore, given their relevant role in immunoregulation, PD- $1^{+}$ILC3s may possibly represent a novel target for therapy in case of pregnancy complications.

In humans, ILCs may be dysregulated in different inflammatory conditions thus representing interesting targets for therapy. ${ }^{25}$ However, to exploit ILCs as targets of disease treatment, we need more information on their functional heterogeneity and plasticity, and on their interactions with other immune and stromal cells in inflammation. Notably, it has been shown that freshly isolated ILC3s from noninflamed tissues express IL-22 transcripts, suggesting that IL-22 is produced under homeostatic conditions. IL-22 
630

production may be important for containment of commensals in gut compartments. ${ }^{26,27}$ On inflammatory stimuli, dendritic cells (DCs) and epithelial cells produce IL-1 $\beta$ and IL-23, inducing secretion of high amounts not only of IL-22 but also of IL-8, GMCSF, and TNFa. These cytokines act on neutrophils, DCs, and other immune cells ensuring better innate protection against pathogens. ${ }^{28}$ Thus, in this context, it is conceivable that the constitutive or inducible expression of inhibitory receptors on innate cells infiltrating decidua may modulate the amplitude and the duration of immune responses and maintain self-tolerance thus preventing collateral tissues damage. Thus, the high expression of TIM-3 and PD-1 on ILC3s could represent a physiological response to the maternal-fetal interface environment and to prevent tissue damage by decidua-infiltrating immune cells.

Clearly, additional studies are needed to precisely dissect the impact of PD-1 and its ligands on ILC3 function. Notably, also mouse and human ILC2s have been shown to express PD-1. ${ }^{29}$ PD- $1^{+}$ILC2s displayed lower proliferative activity after cytokine stimulation, as compared with PD-1 $1^{-}$ILC2s. Importantly, in a model of humanized mice, antibody-mediated blocking of PD-1 restored ILC2 function, both in vitro and in vivo indicating that ILC2s effector functions, and thus type 2 immune responses, can be modulated by PD-1 blocking antibodies. These data provide new clues for therapeutic intervention, allowing to restore type 2 immunity in pregnancy disorders characterized by excessive type 1 immune responses, as result of infections or to other inflammatory events that may occur during the early phases of pregnancy.

In this context, the identification of inhibitory receptors on decidual ILCs and a better understanding of the regulatory activity on ILC functions may offer new clues for a possible use of checkpoint inhibitors in some pregnancy diseases.

\section{MATERIAL AND METHODS}

Isolation of decidual and peripheral blood cells

We obtained 30 decidua samples at 9-12 weeks of gestation from singleton pregnancies of mothers requesting termination of pregnancy for social reasons and 8 placenta samples at 32 -41 weeks of gestation from mothers who have given birth. In both the cases, samples were derived from Policlinico San Martino-IST, Genoa, Italy. Exclusion criteria were HIV and HCV infections. The relevant institutional review boards approved the study and all patients gave their written informed consent according to the Declaration of Helsinki (Delibera $n^{\circ} 625$ ). We isolated cell suspensions from decidual tissue with GentleMacs (Miltenyi Biotec, Bergisch Gladbach, Germany) and cells were then filtered as previously described. ${ }^{30}$ Decidua-infiltrating lymphocytes and peripheral blood mononuclear cells from healthy donor or from pregnant woman were isolated by Fycoll (Cedarlane, Burlington, Ontario, Canada) gradient centrifugation. Decidual infiltrating lymphocytes were analyzed by flow cytometry. Decidual stromal cells (DSC) were isolated and characterized as previously described. ${ }^{10}$

\section{Gating strategy}

Multiparametric flow cytometry analysis of $\mathrm{CD} 45^{+}$decidua lymphocytes (DILs) was performed using a gating strategy (Supplementary Fig. $1 \mathrm{~A}$ ) that allows to identify different ILC subsets. ${ }^{10}$ Subsequently, $\mathrm{CD}^{+} 5^{+}$, Lineage negative ( $\left.\mathrm{Lin}^{-}: \mathrm{CD}^{-}{ }^{-}, \mathrm{CD} 14^{-}, \mathrm{CD}^{-}\right)$cells

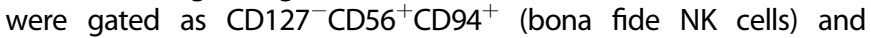
$\mathrm{CD}_{6}{ }^{+} \mathrm{CD} 94^{-} \mathrm{CD} 127^{+} \mathrm{CD} 117^{+}$or $\mathrm{CD}^{-} 6^{-} \mathrm{CD} 94^{-} \mathrm{CD} 127^{+} \mathrm{CD} 117^{+}$ (bona fide ILC3 cells). Decidual ILC3 were further analyzed for the expression of NCRs, in particular NKp46 and NKp44, i.e., markers allowing a more precise identification of ILC3 subsets. The $\mathrm{CD} 56^{+} \mathrm{CD} 94^{-} \mathrm{CD} 127^{+} \mathrm{CD} 117^{+}$subset homogenously expressed NKp44 and high percentages of cells were NKp46 ${ }^{+}$(this subset is referred to as $\left.\mathrm{CD} 56^{+} \mathrm{ILC} 3\right)$. On the other hand, the $\mathrm{CD} 56^{-} \mathrm{CD} 94^{-}$ $\mathrm{CD} 127^{+} \mathrm{CD} 117^{+}$subset expressed low/medium levels of NKp44 and NKp46, depending on the donors (this subset represents the putative LTi-like cells) (Supplementary Fig. 1A). In order to analyze $T$ cells, we gated on $\mathrm{CD} 45^{+} \mathrm{CD} 19^{-} \mathrm{CD} 14^{-} \mathrm{CD} 56^{-} \mathrm{CD} 3^{+}$cells (Supplementary Fig. 1A).

Flow cytometry analyses and monoclonal antibodies

The following mAbs were isolated in our laboratory, licensed to the indicated companies and validated for their specificity: antiNKp30 (AZ20, lgG1), anti-NKp44 (Z231, IgG1), anti-NKp46 (BAB281, lgG1) (Beckman Coulter/Immunotech, Marseille, France). The purified anti-PD-1 mAb (PD1.3.1.3 clone, IgG2b) was originally isolated at the Laboratoire Immunologie des Tumeurs, CRCM, Marseille-Luminy (France). ${ }^{31}$ The following purchased $\mathrm{mAbs}$ were used in this study: Anti-CD56-PC7 (c218 clone), and anti-NKG2A allophycocyanin (Z199 clone) were purchased from Beckman Coulter/Immunotech (Marseille, France); anti-CD3-Viogreen (BW264/56 clone), anti-CD19-VioGreen (LT20 clone), anti-CD14Viogreen (TÜK4 clone), anti-TNF-a-PE, anti-CD223-PE (LAG-3), mAbs were purchased from Miltenyi Biotec (Bergisch Gladbach, Germany); anti-TIM-3 was purchased from Novus Biologicals (Littleton, CO 80120, USA); anti IL-17A-FITC, CD45-APC-H7 (2D1 clone) from BD Bioscience; IL-22-PE TNF-a-eFluor450 purchased from eBioscience (San Diego, CA); CD94-FITC, CD127-BrilliantViolet421, CD117-PerCP-Cy5.5, anti-CRTH2-APC, anti-TIGIT-PE purchased from BioLegend (San Diego, CA); IL-8-PE purchased from R\&D (Minneapolis, MN) and anti-B7-H3 (D-8 clone, lgG2b) was purchased from Santa Cruz Biotechnology (Dallas, TX).

\section{Cytokine production}

For intracellular cytokine detection, freshly isolated decidual lymphocytes were stimulated overnight, as indicated in the result section, with a mixture of cytokines $(50 \mathrm{ng} / \mathrm{ml} \mathrm{IL-23,50} \mathrm{ng/ml} \mathrm{IL-1 \beta}$ and IL-7 (Miltenyi)) plus GolgiStop and GolgiPlug (BD), and $\mathrm{FcR} \gamma^{+} \mathrm{P} 815$ (mastocytoma murine cell line) in the presence or in the absence of anti-NKp46 (BAB281 clone) plus anti-NKp44 (Z231 clone) in combination or not with anti-PD-1 $(1.1 \mu \mathrm{g} / \mathrm{ml}$ ) (or with anti-B7/H3, IgG2B isotype control, $2 \mu \mathrm{g} / \mathrm{ml}$ ) or with anti-TIM-3 mAbs $(2 \mu \mathrm{g} / \mathrm{ml})$ (or with c218, anti-CD56, lgG1 isotype control, $2 \mu \mathrm{g} / \mathrm{ml}$ ). The $E / T$ ratio was $1 / 1$. After stimulation, cells were stained for surface markers, fixed, and permeabilized with Cytofix/Cytoperm (BD) and with Perm/Wash (BD), respectively according to the manufacturer's Instructions. All cytofluorimetric analyses were performed using FACSVerse (Becton Dickinson, Mountain View, CA) and data were analyzed using FlowJo software (TreeStar, Ashland, OR) and FACSuite software version 1.0.3. The P815 cell line has been regularly tested for human cell markers and mycoplasma contamination resulting in all instances negative.

Immunohistochemical analysis

First-trimester decidual samples ( $7 \mu \mathrm{m}$ sections) were formalin-fixed and paraffin-embedded. Samples were de-paraffinized and subsequently exposed to $0.3 \%$ hydrogen peroxide-methanol solution to quench the endogenous peroxidase activity, prior to $30 \mathrm{~min}$ incubation with PD-L1 (27A2 clone IgG2b MBL, Woburn, MA). Then, horseradish peroxidase-conjugated goat anti-human anti-sera (Dako-Envision) and 3-amino-9-ethylcarbazole (Novocastra, Newcastle, UK) were added as previously described. An isotype-matched control mAb was used as negative control. The analysis was performed on 18 samples ( 13 derived from normal decidual samples and 5 from pathological decidual samples). All immunohistochemical evaluations were blindly evaluated by two independent experts.

\section{Statistical analysis}

Statistical analysis was performed with Graphpad Prism (La Jolla, CA) software. For statistical analysis of cytofluorimetric experiments 
(Figs. 1a, c, e, 4a and Supplementary Fig. 4) nonparametric $t$ test (Mann-Whitney test) was used. We performed statistical analysis of cytokine production (Fig. $2 \mathrm{a}-\mathrm{c}$ ) using paired nonparametric $t$ test, Wilcoxon matched-pairs signed rank test. $P$ value of less than 0.05 $\left({ }^{*}\right)$, less than $0.01\left(^{* *}\right)$, less than $0.001\left(^{* * *}\right)$ and less than $0.0001\left(^{* * *}\right)$ was considered statistically significant; when not indicated, data were not statistically significant. The analysis of correlation between CD56 ${ }^{+}$ILC3 or NK and PD-1 expression was analyzed according to a linear regression model (Fig. 4c). Graphs were plotted as mean and standard error of the mean (SEM) or standard deviation (SD).

\section{ACKNOWLEDGEMENTS}

Supported by grants awarded by Associazione Italiana per la Ricerca sul Cancro (AIRC)-Special Program Metastatic disease: the key unmet need in oncology 5 per mille 2018 Id. 21147 (A.M., E.Marcenaro, S.P., M.G., L.M., and P.V.), AIRC IG 2017 Id. 20312 (A.M., E.Marcenaro, S.P., M.G.), AIRC IG 2017 Id. 19920 (L.M., P.V.), Progetto Roche per la Ricerca 2017 (S.P., E.Marcenaro), Ministero della Salute RF-2013, GR2013-02356568 (P.V.). S.P. is recipient of a fellowship awarded by Fondazione Umberto Veronesi. D.O. team was labeled "Equipe FRM DEQ20180339209". D.O. is Senior Scholar of the Institut Universitaire de France.

\section{AUTHOR CONTRIBUTIONS}

S.P. designed and performed research, interpreted data; M.G. performed research, E.F. and E.Munari performed immunohistochemical analysis; D.O. provided important reagents, M.C.M., L.M. and A.M. designed research and wrote the paper, E.Marcenaro and P.V. designed and performed research, interpreted data and wrote the article.

\section{ADDITIONAL INFORMATION}

The online version of this article (https://doi.org/10.1038/s41385-019-0141-9) contains supplementary material, which is available to authorized users.

Competing interests: Daniel Olive is a founder and shareholder of Imcheck Therapeutics (Marseille, France). The remaining authors declare no competing interests.

Publisher's note: Springer Nature remains neutral with regard to jurisdictional claims in published maps and institutional affiliations.

\section{REFERENCES}

1. Diefenbach, A., Colonna, M. \& Koyasu, S. Development, differentiation, and diversity of innate lymphoid cells. Immunity 41, 354-365 (2014).

2. Vivier, E. et al. Innate or adaptive immunity? The example of natural killer cells. Science 331, 44-49 (2011).

3. Marcenaro, E., Dondero, A. \& Moretta, A. Multi-directional cross-regulation of NK cell function during innate immune responses. Transpl. Immunol. 17, 16-19 (2006).

4. Marcenaro, E., Della Chiesa, M., Pesce, S., Agaugue, S. \& Moretta, A. The NK/DC complot. Adv. Exp. Med. Biol. 633, 7-16 (2009).

5. Pesce, S. et al. The innate immune cross talk between NK cells and eosinophils is regulated by the interaction of natural cytotoxicity receptors with eosinophil surface ligands. Front. Immunol. 8, 510 (2017).

6. Riise, R. E. et al. TLR-stimulated neutrophils instruct NK cells to trigger dendritic cell maturation and promote adaptive T cell responses. J. Immunol. 195, 1121-1128 (2015).

7. Hoorweg, K. et al. Functional differences between human NKp44(-) and NKp44 (+) RORC(+) innate lymphoid cells. Front. Immunol. 3, 72 (2012).
8. Moretta, A. et al. Receptors for HLA class-I molecules in human natural killer cells. Annu. Rev. Immunol. 14, 619-648 (1996).

9. Pesce, S. et al. Identification of a subset of human natural killer cells expressing high levels of programmed death 1: A phenotypic and functional characterization. J. Allergy Clin. Immunol. 139, 335-346 e333 (2017).

10. Vacca, P. et al. Identification of diverse innate lymphoid cells in human decidua. Mucosal Immunol. 8, 254-264 (2015).

11. Vacca, P., Moretta, L., Moretta, A. \& Mingari, M. C. Origin, phenotype and function of human natural killer cells in pregnancy. Trends Immunol. 32, 517-523 (2011).

12. Mjosberg, J. M. et al. Human IL-25- and IL-33-responsive type 2 innate lymphoid cells are defined by expression of CRTH2 and CD161. Nat. Immunol. 12, 1055-1062 (2011).

13. Kitaya, K. et al. IL-15 expression at human endometrium and decidua. Biol. Reprod. 63, 683-687 (2000).

14. Croxatto, D. et al. Group 3 innate lymphoid cells regulate neutrophil migration and function in human decidua. Mucosal Immunol. 9, 1372-1383 (2016).

15. Keir, M. E., Butte, M. J., Freeman, G. J. \& Sharpe, A. H. PD-1 and its ligands in tolerance and immunity. Annu. Rev. Immunol. 26, 677-704 (2008).

16. $\mathrm{Xu}, \mathrm{Y}$. et al. Innate lymphoid cells at the human maternal-fetal interface in spontaneous preterm labor. Am. J. Reprod. Immunol. 79, e12820 (2018).

17. King, A. et al. Recognition of trophoblast HLA class I molecules by decidual NK cell receptors-a review. Placenta 21(Suppl A), S81-S85 (2000).

18. Hackmon, R. et al. Definitive class I human leukocyte antigen expression in gestational placentation: HLA-F, HLA-E, HLA-C, and HLA-G in extravillous trophoblast invasion on placentation, pregnancy, and parturition. Am. J. Reprod. Immunol. 77, e12463 (2017).

19. Vacca, P. et al. Crosstalk between decidual NK and CD14 + myelomonocytic cells results in induction of Tregs and immunosuppression. Proc. . Natl. Acad. Sci. USA 107, 11918-11923 (2010).

20. Li Y., Li D. \& Du M. TIM-3: a crucial regulator of NK cells in pregnancy. Cell. Mol. Immunol. 14, 948-950 (2017).

21. Sun, J. et al. Tim-3 is upregulated in NK cells during early pregnancy and inhibits NK cytotoxicity toward trophoblast in galectin-9 dependent pathway. PLOS ONE 11, e0147186 (2016).

22. Meggyes, M. et al. Feto-maternal immune regulation by TIM-3/galectin- 9 pathway and PD-1 molecule in mice at day 14.5 of pregnancy. Placenta 36, 1153-1160 (2015).

23. Wang, S. C. et al. PD-1 and Tim-3 pathways are associated with regulatory CD8 + T-cell function in decidua and maintenance of normal pregnancy. Cell Death Dis. 6, e1738 (2015).

24. Nowak, I. et al. KIR, LILRB and their Ligands' Genes as Potential Biomarkers in Recurrent Implantation Failure. Arch. Immunol. Ther. Exp. (Warsz.) 65, 391-399 (2017).

25. Glatzer, T. et al. RORgammat $(+)$ innate lymphoid cells acquire a proinflammatory program upon engagement of the activating receptor NKp44. Immunity 38, 1223-1235 (2013).

26. Hanash, A. M. et al. Interleukin-22 protects intestinal stem cells from immunemediated tissue damage and regulates sensitivity to graft versus host disease. Immunity 37, 339-350 (2012).

27. Sonnenberg, G. F. et al. Innate lymphoid cells promote anatomical containment of lymphoid-resident commensal bacteria. Science 336, 1321-1325 (2012)

28. Hazenberg, M. D. \& Spits, H. Human innate lymphoid cells. Blood 124, 700-709 (2014).

29. Taylor, S. et al. PD-1 regulates KLRG1 $(+)$ group 2 innate lymphoid cells. J. Exp. Med. 214, 1663-1678 (2017).

30. Vacca, P. et al. Analysis of natural killer cells isolated from human decidua: Evidence that 2B4 (CD244) functions as an inhibitory receptor and blocks NK-cell function. Blood 108, 4078-4085 (2006).

31. Ghiotto, M. et al. PD-L1 and PD-L2 differ in their molecular mechanisms of interaction with PD-1. Int. Immunol. 22, 651-660 (2010). 